\title{
Reduction of ADAMTS13 Levels Predicts Mortality in SARS-CoV-2 Patients
}

\author{
Giovanni L. Tiscia ${ }^{1}$ Giovanni Favuzzi ${ }^{1}$ Antonio De Laurenzo ${ }^{1}$ Filomena Cappucci ${ }^{1}$ Lucia Fischetti ${ }^{1}$ \\ Lazzaro di Mauro $^{2}$ Giuseppe Miscio ${ }^{2}$ Antonio Mirijello ${ }^{30}$ Elena Chinni ${ }^{1}$ Elvira Grandone ${ }^{10}$ \\ on behalf of CSS COVID-19 Group*
}

\footnotetext{
${ }^{1}$ Research Unit of Thrombosis and Hemostasis, Fondazione IRCCS "Casa Sollievo della Sofferenza," San Giovanni Rotondo, Italy

2 Transfusion Medicine and Laboratory Department, Fondazione IRCCS "Casa Sollievo della Sofferenza," San Giovanni Rotondo, Italy

3 Medical Department, Fondazione IRCCS "Casa Sollievo della

Sofferenza," San Giovanni Rotondo, Italy
}

TH Open 2020;4:e203-e206.

In SARS-CoV-2 (severe acute respiratory syndrome coronavirus 2) infected patients, hemostasis shows an uncontrolled activation, with a high D-dimer concentration. ${ }^{1,2}$ It has been also shown that the SARS-CoV-2 can promote endotheliitis, ${ }^{3}$ and in turn, pulmonary endothelial damage fostering thrombogenesis. ${ }^{4}$

Thrombotic microangiopathy (TMA) is defined by microangiopathic hemolytic anemia, thrombocytopenia, and organ failure ${ }^{5}$ and can be observed in several disorders. ${ }^{6}$ Infectious diseases could be one of these disorders. ${ }^{7} \mathrm{~A}$ disintegrin and metalloproteinase with thrombospondin motifs 13 (ADAMTS13) plays a pathophysiological role in the TMA, including that arousing on sepsis. ${ }^{8}$ Moreover, a key role of the ADAMTS13 in the coagulopathy of sepsis is also acknowledged. ${ }^{9}$ In the same scenario, ADAMTS13 was shown to be one of the endothelium-related markers showing changes, with a trend toward a reduction. ${ }^{10}$ Furthermore, in response to the endothelial activation-induced vascular inflammation, reciprocal changes of the ADAMTS13 and its substrate, the von Willebrand factor (vWF), are observed. ${ }^{11}$

In septic patients, reduced synthesis of protein C (PC) and its cofactor protein $\mathrm{S}$ (PS) in addition to impaired expression of thrombomodulin and endothelial PC receptor on endothelium determines reduced PC levels with an imbalance of thrombin regulation. ${ }^{12}$ Furthermore, antithrombin (AT)the most important inhibitor of thrombin and activated factor $\mathrm{X}$-is reduced as a consequence of its consumption and impaired synthesis and degradation. ${ }^{13}$

We aimed at assessing whether ADAMTS13 levels predict mortality in patients with a diagnosis of SARS-CoV-2 infection.

Members of the CSS COVID-19 Group are provided in the Supplementary Material.
Address for correspondence Elvira Grandone, MD, PhD, Fondazione I. R.C.C.S. "Casa Sollievo della Sofferenza," Research Unit of Thrombosis and Hemostasis, 71013 San Giovanni Rotondo (Foggia), Italy (e-mail: e.grandone@operapadrepio.it).

The secondary aim was to investigate a possible relationship between ADAMTS13, D-dimer, vWF, and natural anticoagulants levels to have easy-to-handle markers of clinical outcomes. Seventy-seven patients admitted between March 1 and April 30, 2020 at the "Casa Sollievo della Sofferenza" Research Hospital with a laboratory-confirmed diagnosis (i.e., RT-PCR according to the protocol established by the $\mathrm{WHO})^{14}$ of the SARS-CoV-2 infection were investigated. Demographic and clinical information and routine laboratory data at the hospital admission were extracted from hospital electronic medical records. In all patients, ADAMTS13, vWF antigen (vWF:Ag)/ functional, and natural anticoagulants (AT, PC, and PS) levels were measured on a blood sample obtained within seven hospitalization days. The ADAMTS13 activity levels were measured using a chromogenic enzyme-linked immunosorbent assay (ELISA) method (TECHNOZYM ADAMTS13 Activity ELISA Kit, Technoclone, Austria). Continuous variables are presented as a median and interquartile range, whereas discrete variables as numbers and percentage. After testing for data normality distribution, Mann-Whitney test was used to analyze differences in continuous variables. Fisher's exact test was also performed to estimate the association between D-dimer positive test ( $>500 \mathrm{ng} / \mathrm{mL}$ ) and ADAMTS13 activity levels below median value. Spearman's $r$ was used to explore the reciprocal relationship between ADAMTS13 and D-dimer, vWF, and natural anticoagulants: results are given as $r$ - and $p$ values. Multiple linear regression analysis, which controlled for D-dimer concentration, age, and sex was performed to evaluate a predictive model for ADAMTS13 activity levels. Kaplan-Meier analysis was performed to assess whether ADAMTS13 can predict mortality. Statistical significance was set at $p$-values $<0.05$. All the analyses were performed using GraphPad Prism version 8.00 for Windows, GraphPad Software

(c) 2020 Georg Thieme Verlag KG License terms

DOI https://doi.org/ 10.1055/s-0040-1716379. ISSN 2512-9465.
Stuttgart · New York

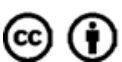


(La Jolla, California, United States, www.graphpad.com). The study was approved by the local ethics committee and was performed according to the Declaration of Helsinki on Ethical Principles for medical research involving human subjects. All patients gave written informed consent.

Descriptive analysis is shown in - Table 1. Most patients (73\%) were older than 60 years, with a slightly higher percentage (56 vs. $46 \%$ ) of women. Almost all the patients

Table 1 Demographic/clinical information and laboratory data collected in the study population

\begin{tabular}{|c|c|}
\hline Variables & Values \\
\hline \multicolumn{2}{|l|}{ Demographic and clinical information } \\
\hline Age, median (IQR) & $61.5(80-62)$ \\
\hline Female/Male & $43 / 34$ \\
\hline \multicolumn{2}{|l|}{ Coexisting morbidities, $n$ (\%) } \\
\hline Any & $22(28.5)$ \\
\hline Hypertensive disorder & $17(22)$ \\
\hline Chronic renal disease & $8(10.4)$ \\
\hline Diabetes & $8(10.3)$ \\
\hline Dilated cardiomyopathy & $7(9)$ \\
\hline Chronic obstructive chronic disease & $7(9)$ \\
\hline Atrial fibrillation & $6(7.8)$ \\
\hline None & $2(3)$ \\
\hline Deaths, $n(\%)$ & $4(5.2)$ \\
\hline \multicolumn{2}{|l|}{ Laboratory data } \\
\hline $\begin{array}{l}\text { ADAMTS13 activity }(\mathrm{U} / \mathrm{dL}) \text {, } \\
\text { median (IQR) }\end{array}$ & $70(80-60)$ \\
\hline vWF:Ag (\%), median (IQR) & $231.2(415.3-205.7)$ \\
\hline vWF:RCo (\%), median (IQR) & $150.0(334.3-116.9)$ \\
\hline ADAMTS13/vWF:RCo, median (IQR) & $0.40(0.50-0.23)$ \\
\hline vWF:RCo/vWF:Ag, median (IQR) & $0.65(0.87-0.6)$ \\
\hline Factor VIII (\%), median (IQR) & $95.20(133.0-69.40)$ \\
\hline D-dimer (ng/mL) & $882.0(2250-460.0)$ \\
\hline Hemoglobin (g/dL), median (IQR) & $11.9(13.3-9.8)$ \\
\hline $\begin{array}{l}\text { Platelet count }\left(\times 10^{9} / \mathrm{L}\right) \text {, } \\
\text { median (IQR) }\end{array}$ & $234(283-149)$ \\
\hline $\begin{array}{l}\text { White cell count }\left(\times 10^{9} / \mathrm{L}\right) \text {, } \\
\text { median (IQR) }\end{array}$ & $6.1(9.4-4.5)$ \\
\hline $\begin{array}{l}\text { Lactate dehydrogenase }(\mathrm{U} / \mathrm{L}) \text {, } \\
\text { median (IQR) }\end{array}$ & $236.0(314.5-197.0)$ \\
\hline Creatinine (mg/dL), median (IQR) & $0.9(1.5-0.6)$ \\
\hline $\begin{array}{l}\text { C-reactive protein (mg/L), } \\
\text { median (IQR) }\end{array}$ & $1.7(9.3-0.4)$ \\
\hline $\begin{array}{l}\text { Erythrocyte sedimentation } \\
\text { rate }(\mathrm{mm} / \mathrm{h})\end{array}$ & $34(79.5-23.5)$ \\
\hline $\begin{array}{l}\text { Prothrombin time (International } \\
\text { Normalized Ratio), median (IQR) }\end{array}$ & $1.09(1.18-1.06)$ \\
\hline $\begin{array}{l}\text { Activated partial thromboplastin } \\
\text { time }(\mathrm{s}) \text {, median (IQR) }\end{array}$ & $25.0(26.5-23.0)$ \\
\hline
\end{tabular}

Abbreviations: ADAMTS13, a disintegrin and metalloproteinase with thrombospondin motifs 13; IQR, interquartile range; vWF:Ag, von Willebrand factor antigen; vWF:RCo, von Willebrand factor ristocetin cofactor.
(97.5\%) showed comorbidities. Fourteen patients (18\%) were admitted to the intensive care unit. Median value of ADAMTS13 in the entire sample was $70 \mathrm{U} / \mathrm{dL}$. Kaplan-Meier analysis showed a significantly lower survival in those individuals with ADAMTS13 activity below the median value $(p=0.025)$ ( - Fig. 1). Overall, we recorded four deaths (5.2\%), all in patients with hypertension, two of them with associated ischemic cardiomyopathy (one diabetic). We cannot exclude that comorbidities could have contributed to mortality in these patients. Seventy-three patients were discharged $(n=51)$ or are still alive $(n=22)$ at the end of the study. Since vWF might be directly involved in the pathophysiology of TMA, we performed also the Kaplan-Meier analysis according to vWF ristocetin cofactor (vWF:RCo) levels: no statistically significant difference was found, in terms of mortality prevalence $(p=0.80)$. Multiple linear regression showed that $\mathrm{D}$-dimer concentration at admission $(p=0.0145)$ and age $(p=0.0036)$ were independently associated with ADAMTS13 activity levels. D-dimer concentration above $500 \mathrm{ng} / \mathrm{mL}$ at admission was associated with a 2.8fold higher likelihood of ADAMTS13 activity below the median value (odds ratio: 4.8 [95\% confidence interval: 1.3-16.9], $p=0.02$ ). Lastly, D-dimer concentration, vWF: $\mathrm{Ag}$, and natural anticoagulants (AT, PC, and PS) levels were inversely (D-dimer and vWF) or directly (AT, PC, PS) associated with ADAMTS13 levels (Supplementary Table S1). ADAMTS13 activity negatively correlated with D-dimer concentration (Spearman's $r$ : $-0.41, p=0.002$ ) and vWF:Ag (Spearman's $r$ : $-0.34, p=0.005$ ) levels, whereas a positive correlation was found with AT (Spearman's $r$ : 0.45 , $p<0.001$ ), PC (Spearman's $r: 0.33, p=0.004$ ), and PS (Spearman's $r: 0.23, p=0.04)$. The main finding of this study is that patients with ADAMTS13 activity below $70 \mathrm{U} / \mathrm{dL}$ have a higher risk of in-hospital death. Present findings are consistent with those observed in patients with sepsis, in whom low ADAMTS13 levels are inversely correlated with $\mathrm{vWF}^{15}$ and a poor prognosis, as well as with sepsis severity. ${ }^{16,17}$ Elevated levels of vWF and reduced ADAMTS13 could correlate with endothelial damage and a progressive worsening of the organ function. In our setting, the ratio of vWF:RCo/vWF: $\mathrm{Ag}$ is 0.65 . This value does not reflect a predominance of ultra-large vWF multimers, which could represent the culprit of microangiopathic thrombosis. We hypothesize that, at variance with other TMA, the underlying pathogenetic mechanism is the endothelial inflammation and damage resulting in a slight reduction of ADAMTS13 levels. Consistent with this possible pathogenetic mechanism is the lack of thrombocytopenia. Indeed, in TMA other than thrombotic thrombocytopenic purpura, the relationship between reduced ADAMTS13 activity and thrombocytopenia is not clearly demonstrated. ${ }^{18}$ As reported in other septic patients, the endothelial damage triggers the coagulation cascade, as suggested by elevated D-dimer and decreased natural anticoagulants plasma levels. ${ }^{19,20}$ Notably, we observed that Ddimer, a blood parameter routinely investigated in SARSCoV-2 patients for the possible clinical impact on mortality in these settings, ${ }^{21,22}$ strongly predicts ADAMTS13 levels. Thus, it is conceivable that endothelial dysfunction could be 


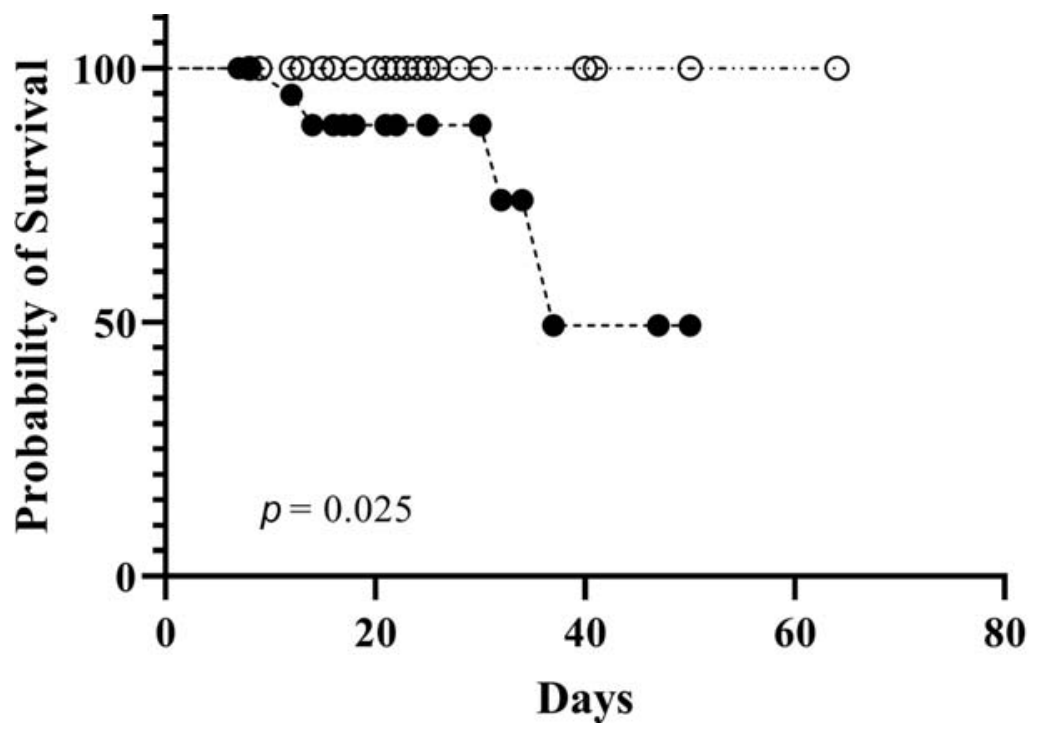

-๑. ADAMTS13 $\geq 70 \mathrm{U} / \mathrm{dL}$

- ADAMTS $13<70 \mathrm{U} / \mathrm{dL}$

Fig. 1 Kaplan-Meier survival analysis according to a disintegrin and metalloproteinase with thrombospondin motifs 13 (ADAMTS13) activity levels: four deaths were observed in the group with values $<70 \mathrm{U} / \mathrm{dL}$.

reflected in any of the hemostasis phases and variables, including the natural anticoagulants. Endothelial dysfunction is a key finding and major determinant of poor prognosis in SARS-CoV-2 patients. ${ }^{23}$ Indeed, direct or indirect activation by SARS-CoV-2 on endothelium would cause intensive vWF secretion from Weibel-Palade bodies, as expected upon perturbations on these vascular cells. ${ }^{13}$ Our data are consistent with previous findings suggesting that SARS-CoV-2 patients present a low-grade disseminated coagulopathy and localized pulmonary microangiopathy, resulting in organ dysfunction. ${ }^{24}$ Notwithstanding the retrospective nature and limited size of this study, overall, present findings may speculatively suggest that ADAMTS13 would represent a clinic-diagnostic marker predicting SARS-CoV-2-related prognosis.

\section{Authors' Contributions}

G.L.T.: analysis of data and drafting the article; G.F. and E. C.: analysis and interpretation of data; A.D.L.: collection and management of data; F.C. and L.F.: laboratory investigations; L.d.M. and G.M.: collection of data; A.M.: selection of patients; E.G.: conception and design of study, revising the article critically for important intellectual content. All authors critically revised the article and approved the final version.

\section{Conflict of Interest}

None declared.

\section{References}

1 Connors JM, Levy JH. COVID-19 and its implications for thrombosis and anticoagulation. Blood 2020;135(23):2033-2040

2 Tang N, Li D, Wang X, Sun Z. Abnormal coagulation parameters are associated with poor prognosis in patients with novel coronavirus pneumonia. J Thromb Haemost 2020;18(04):844-847

3 Varga Z, Flammer AJ, Steiger P, et al. Endothelial cell infection and endotheliitis in COVID-19. Lancet 2020;395(10234): 1417-1418
4 Marini JJ, Gattinoni L. Management of COVID-19 respiratory distress. JAMA [Internet] 2020. Doi: 10.1001/jama.2020.6825

5 Moake JL. Thrombotic microangiopathies. N Engl J Med 2002;347 (08):589-600

6 George JN, Nester CM. Syndromes of thrombotic microangiopathy. N Engl J Med 2014;371(07):654-666

7 Coppo P, Adrie C, Azoulay E, et al. Infectious diseases as a trigger in thrombotic microangiopathies in intensive care unit (ICU) patients? Intensive Care Med 2003;29(04):564-569

8 Masias C, Cataland SR. The role of ADAMTS13 testing in the diagnosis and management of thrombotic microangiopathies and thrombosis. Blood 2018;132(09):903-910

9 Levi M, Scully M, Singer M. The role of ADAMTS-13 in the coagulopathy of sepsis. J Thromb Haemost 2018;16(04):646651

10 Vasileiadis I, Politou M, Dimopoulos S, et al. Variation of endothelium-related hemostatic factors during sepsis. Microcirculation 2018;25(08):e12500

11 Chen J, Chung DW. Inflammation, von Willebrand factor, and ADAMTS13. Blood 2018;132(02):141-147

12 Levi M, van der Poll T. Inflammation and coagulation. Crit Care Med 2010;38(2, Suppl):S26-S34

13 Keller TT, Mairuhu AT, de Kruif MD, et al. Infections and endothelial cells. Cardiovasc Res 2003;60(01):40-48

14 World Health Organization. Coronavirus disease (COVID-19) technical guidance: laboratory testing for 2019-nCoV in humans. Available at: https://www.who.int/emergencies/diseases/novelcoronavirus-2019/technical-guidance/laboratory-guidance. Accessed August 12, 2020

15 Kremer Hovinga JA, Zeerleder S, Kessler P, et al. ADAMTS-13, von Willebrand factor and related parameters in severe sepsis and septic shock. J Thromb Haemost 2007;5(11):2284-2290

16 Martin K, Borgel D, Lerolle N, et al. Decreased ADAMTS-13 (a disintegrin-like and metalloprotease with thrombospondin type 1 repeats) is associated with a poor prognosis in sepsis-induced organ failure. Crit Care Med 2007;35(10):2375-2382

17 Lin JJ, Chan OW, Hsiao HJ, et al. Decreased ADAMTS 13 activity is associated with disease severity and outcome in pediatric severe sepsis. Medicine (Baltimore) 2016;95(16):e3374

18 Song J, Lee KA, Park TS, Park R, Choi JR. Linear relationship between ADAMTS13 activity and platelet dynamics even before severe thrombocytopenia. Ann Clin Lab Sci 2008;38(04): 368-375 
19 Dhainaut J-F, Shorr AF, Macias WL, et al. Dynamic evolution of coagulopathy in the first day of severe sepsis: relationship with mortality and organ failure. Crit Care Med 2005;33(02):341-348

20 Mathieu J, Boucher S. Sepsis-associated coagulopathy: diagnosis with hematologic biomarkers and a novel hemostatic score that may predict the risk of developing a multiple organ dysfunction syndrome and a higher rate of mortality. Chest 2015;148:340A

21 Zhou F, Yu T, Du R, et al. Clinical course and risk factors for mortality of adult inpatients with COVID-19 in Wuhan, China: a retrospective cohort study. Lancet 2020;395(10229):1054-1062
22 Zhang L, Yan X, Fan Q et al. D-dimer levels on admission to predict in-hospital mortality in patients with Covid-19. J Thromb Haemost 2020;18(06):1324-1329

23 Sardu C, Gambardella J, Morelli MB, Wang X, Marfella R, Santulli G. Hypertension, thrombosis, kidney failure, and diabetes: is COVID19 an endothelial disease? A comprehensive evaluation of clinical and basic evidence. J Clin Med 2020;9(05):1417

24 Levi M, Thachil J, Iba T, Levy JH. Coagulation abnormalities and thrombosis in patients with COVID-19. Lancet Haematol 2020;7 (06):e438-e440 